\title{
X-ray structural studies of the Pd-In-Ru system using synchrotron radiation
}

\author{
O. V. Akimova ${ }^{1}$, A. A. Veligzhanin ${ }^{2}$ \\ ${ }^{1}$ Moscow State University,Moscow, 119991 Russia \\ ${ }^{2}$ National Researches Centre "Kurchatov Institute", Moscow, 123098 Russia \\ akimova@physics.msu.ru
}

PACS 61.10.-i, 64.60.Cn, 61.82.Bg

DOI 10.17586/2220-8054-2018-9-6-741-745

\begin{abstract}
The paper presents X-ray diffraction studies of diffusion filters-membranes based on palladium. Information on the structural state of membranes was obtained using synchrotron radiation (SR) of Kurchatov Synchrotron Radiation Source. A comparison was made between states of the prolonged membrane relaxation after hydrogenation and that exposed to additional applied load by the method of electrolytic hydrogenation $(\mathrm{EH})$. In addition to the structural maxima determining the face-centered lattice of the alloy matrix, reflections were revealed on the diffraction patterns, indicating the presence of long-range ordering in the structure.
\end{abstract}

Keywords: X-ray diffraction, synchrotron radiation, superstructure, palladium-based alloys.

Received: 15 October 2018

Revised: 27 October 2018

\section{Introduction}

When metal filters are used in the diffusion of high purity hydrogen production [1-4], the problems of reliability and duration of the membrane operation arise during interaction with hydrogen $[4,5]$. These issues are related to changes in the structure of metals and alloys as a result of their hydrogenation [5-8]. Therefore, $\mathrm{X}$-ray structural studies for analysis of the kinetics of structural defects in diffusion filters after hydrogenation are needed for the development of safe industrial technologies for production $[1,4]$, storage and transfer of high-purity hydrogen $[2,3]$.

Atoms of alloying elements added to palladium in order to improve the mechanical characteristics and reliability of membrane operation in an aggressive gaseous medium [4]. The additives usually form point defects in the lattice of the metal-solvent that can be ordered to some degree. As a rule, hydrogenation is associated with strong plastic deformation for membranes, which is accompanied by the appearance and development of order-disorder processes in the distribution of metal atoms $[9,10]$. Hydrogenation enhances diffusion processes $[8,11]$ and initiates phase transformations [12-14], generating a high concentration of vacancies, especially for various chemical interactions or affinity of elements with hydrogen $[8,15,16]$. Phase transformations in hydrogenated systems occur over a period of time [13] and affect on many of the structure-sensitive properties of membranes $[1,4,5]$.

There are several advantages for synchrotron radiation (SR) in comparison with X-ray tube radiation, including: brightness, high photon flux, level of monochromatization, easily adjusted X-ray wavelength, a high degree of polarization and collimation provide a new level of X-ray analysis as well as the quality of information acquisition [17-19]. Therefore, the aim of this study was to perform an X-ray diffraction study of membranes using SR with special attention to the region of small angles where order-disorder phase transitions occur in the Pd-In-Ru system after hydrogenation can be observed.

The X-ray experiment was carried out using the equipment of the "Structural Material Science" station of Kurchatov Synchrotron Radiation Source [20].

\section{Experimental and calculation details}

\subsection{The object of research}

Foils Pd-6 wt.\% In-0.5 wt.\% Ru were developed and prepared by the research group of the Institute of Metallurgy and Material Science of the Russian Academy of Sciences (IMET RAN) [4]. The membranes of given composition possess high hydrogen permeability, good corrosion resistance, high plasticity and strength during maintenance in hydrogen containing gas mixtures. Nevertheless, there are some problems with their reliability that can be related with structural changes in membranes occurring during their operation in hydrogen.

Foils of $50 \mu \mathrm{m}$ thickness were fabricated from high purity materials $(\approx 99.9 \%)$ by electric arc melting and cold rolling of blanks on a four-roll rolling mill with intermediate vacuum annealing at a temperature of $900{ }^{\circ} \mathrm{C}$ for 1 hour [4]. Further, the foils were used as a filter in the plant for the determination of hydrogen permeability [19]. 
Then, the membranes, denoted as foil 1 and foil 2, were cooled slowly to the room temperature and stored under normal conditions (room temperature and pressure) for a year.

Further, foil 2 was electrolytically saturated by hydrogen (electrolyte- $4 \%$ aqueous solution Na-F) during 0.5 hour at a current density of $10 \mathrm{~mA} / \mathrm{cm}^{2}$. X-ray diffraction (XRD) patterns were obtained using $\mathrm{Cu}-\mathrm{K} \alpha_{1}$ radiation upon the Bragg-Brentano geometry $[13,14]$. X-ray diffractograms were measured over a narrow angular range near the structural maxima corresponding to the "main" phase of the foil. Only the upper $6 \mu \mathrm{m}$-thick layer of the foil contributed to the diffraction maxima.

\subsection{X-ray experiment using synchrotron radiation (SR)}

The X-ray diffraction with synchrotron radiation source was carried out in the Debye transmission geometry. A beam of SR was monochromatized using a silicon 111 channel-cut monochromator with energy resolution of $2 \times 10^{-4} \mathrm{keV}$. The beam size was determined using motorized slits and was equal to $(250 \times 250) \mu \mathrm{m}^{2}$. The diffraction patterns of the samples were obtained at $0.6199 \AA$ and $0.4573 \AA$, corresponding to the energies $20 \mathrm{keV}$ and $24 \mathrm{keV}$. The samples were oriented perpendicularly to the beam. The scattering radiation was recorded by a two-coordinate detector Mar CCd165. During measurement, the samples performed an oscillatory motion perpendicular to the beam SR in two directions: within a range of $4 \mathrm{~mm}$ in the horizontal direction and $1 \mathrm{~mm}$ in the vertical direction, thus improving the averaging over crystallites orientations. To determine the distance and non-orthogonality of the detector to the beam, a standard sample of $\mathrm{CeO}_{2}$ was used. Two dimensional X-ray diffraction patterns were integrated to one-dimensional I $(2 \theta)$ curves by Fit2D software [21]. Analysis of diffraction profiles was carried out using the software package "Fityk" [22].

The phase diagram of the Pd-In-Ru system is not available in the literature. Since the concentration of Ru in the samples is small, and the difference between the atomic radii of Pd and Ru is about $2 \%(1.37 \AA$ and $1.34 \AA$ respectively) the system was considered binary. The Pd-In phase diagram [23] shows that solid solution Pd-6 wt.\% In at room temperature is in single-phase region (Fig. 1). Indium concentration in observed phases was determined from lattice parameters according to Vegard law.

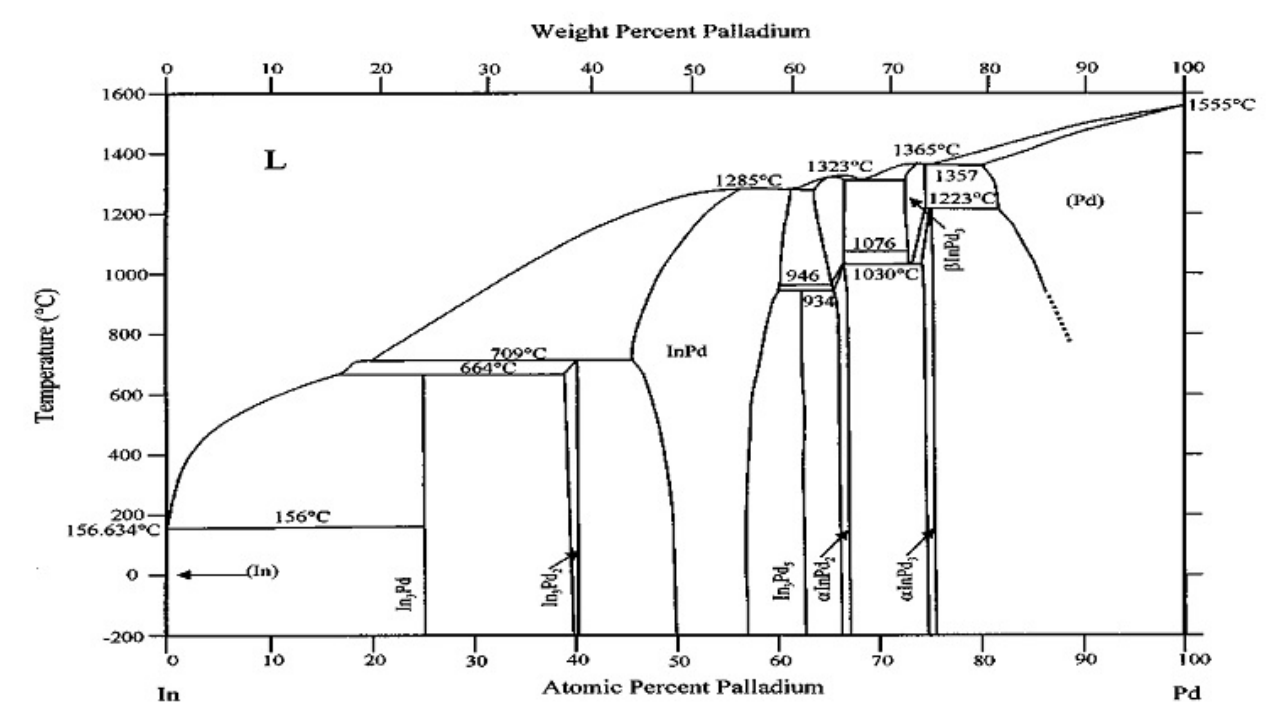

FIG. 1. Phase diagram of Pd-In system [23]

\section{Results and discussion}

Diffraction patterns of foil 1 and foil 2 obtained at the radiation energy $20 \mathrm{keV}$ are shown onto Fig. 2. The angular positions of intense diffraction maxima corresponding to the main phase of foil 1 have determined a facecentered cubic lattice with a period $a_{0}=3.9031 \pm 0.0019 \AA$. The concentration of In was estimated to be $4.87 \pm 0.04$ at. \%. For foil 2, a face-centered cubic lattice with a period $a_{0}=3.9043 \pm 0.0012$ at. $\%$ has determined similarly, which corresponds to the In concentration of about $5.32 \pm 0.02$ at.\%.

Differences in the intensity of near orders reflections of coherent scattering regions (CSR) with Miller indices 111 and 100, in further CSR (111) and CSR (100), at identical relation of intensities for the far order reflections both foils may be showing the increasing effect of extinction upon the crystallographic direction [111]. 
In addition to the maxima corresponding to the main phase of the membranes, diffraction peaks of low intensity were found. The phenomenon of such reflections on diffraction patterns indicated in the membranes the presence of areas with concentrations of In significantly different from the alloy matrix. As one can see from the diffractograms in Fig. 2, the diffraction maxima of such phases were found in diffractograms of both foils and had a similar shape.

For foil 1, the diffraction peak isolated near the main maximum for CSR (110) (Fig. 2(a)) has corresponded to the phase with the lattice parameter $4.0868 \pm 0.0012 \AA$. Near it there is maximum that has corresponded for the basis phase with the lattice parameter $3.9059 \pm 0.0004 \AA$. That result corresponded to a concentration $5.9 \pm 0.1$ at. $\%$ In.

For foil 2, a similar diffraction peak near the main maximum for CSR (110) (Fig. 2(b)) has allowed to identify three phases with lattice parameters $4.0731 \pm 0.0008 \AA, 4.0826 \pm 0.0011 \AA, 4.0912 \pm 0.0009 \AA$. Also for foil 2 , a small fraction phase with lattice parameter $3.9577 \pm 0.0004 \AA$, the In content is $25.3 \pm 0.1$ at. \%, was determined for CSR (111).

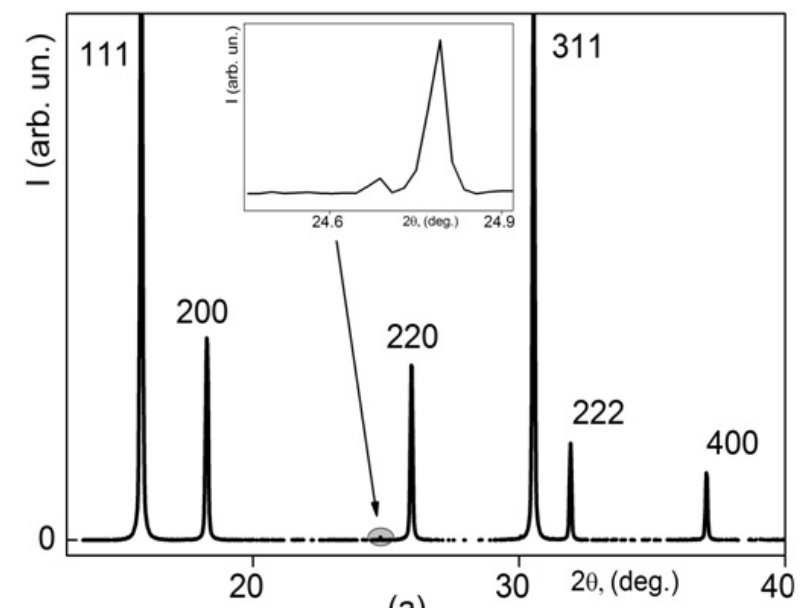

(a)

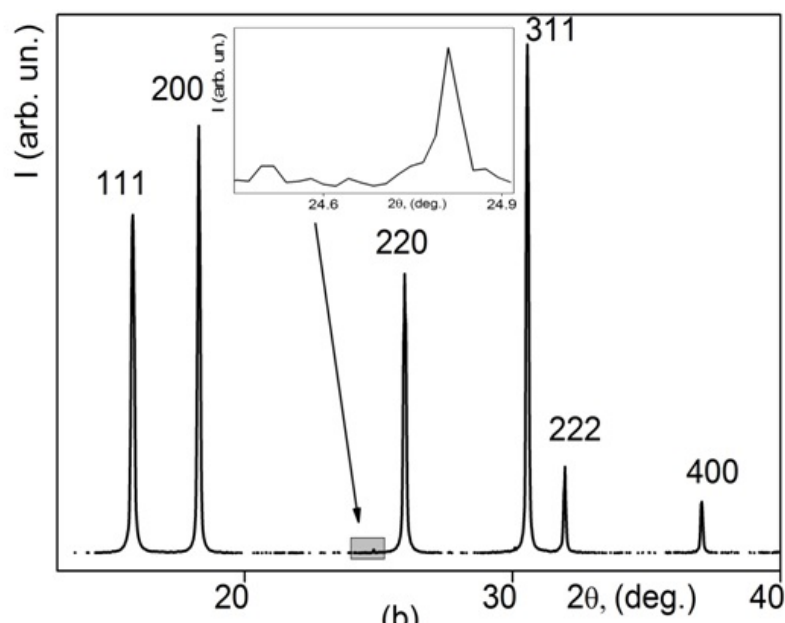

(b)

FIG. 2. Diffraction patterns foils at a beam energy of SR $20 \mathrm{keV}$ : (a) - for foil 1; (b) - for foil 2

In order to investigate the possible superstructure nature of these phases, the diffraction spectra of foils were obtained at a beam energy of SR $24 \mathrm{keV}$ (Fig. 3).

Structural reflections for the base (main) phase have revealed a cubic face-centered lattice for both foils with the same parameters as specified above.

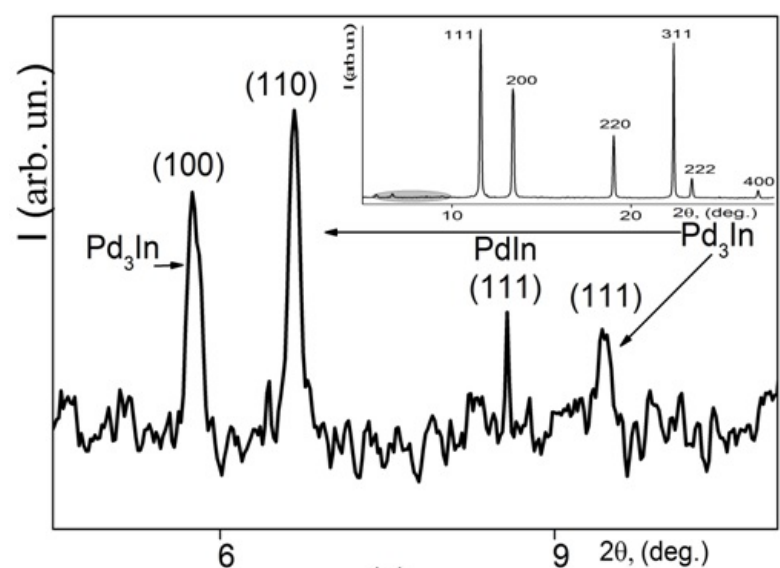

(a)

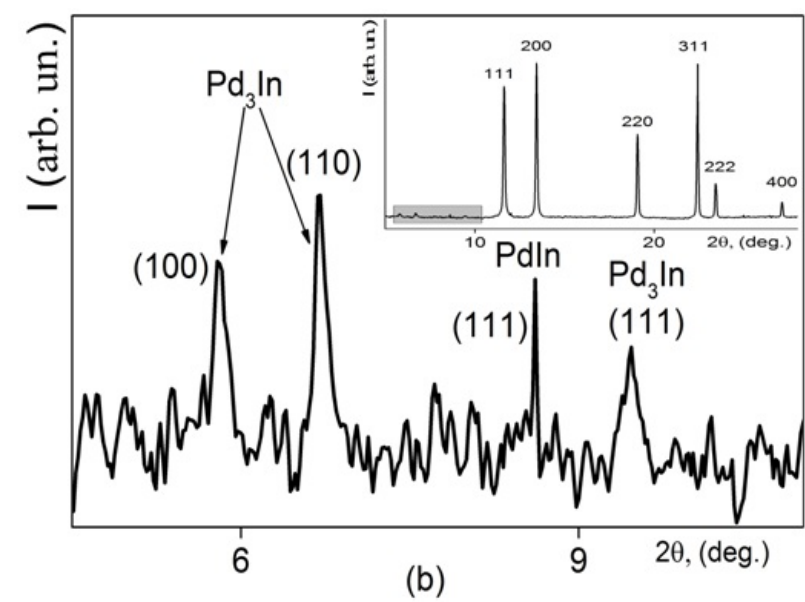

(b)

FIG. 3. Diffraction patterns foils at a beam energy of SR $24 \mathrm{keV}$, the definition superstructure regions: (a) - for foil 1 ; (b) - for foil 2 
Finding reflections with prohibited interference indices for a face-centered cubic cell have indicated the presence of long-range ordering regions in both foils [24,25]. Marked on Fig. 3, the diffraction peaks of low intensity correspond to the first order reflections for CSR (100), CSR (111) and CSR (110).

The results of the research show that the beginning of the process of regularization is likely upon the first hydrogenation of foils in IMET [4]. Differences in the shape of profiles similar superstructural maxima indicate that electrolytic hydrogenation can make some adjustments into the distribution of the components of the superstructural inclusions.

The angular positions of the superstructural peaks show phases corresponding to two groups with the formulas $\mathrm{Pd}_{3}$ In and PdIn. The results obtained are in agreement with the phase diagram data (Fig. 1).

The analysis of the angular positions of diffraction maxima corresponding to the phase $\mathrm{Pd}_{3} \mathrm{In}$ revealed that the phase has a face-centered lattice. That is, the ordering process in the arrangement of atoms has passed with the preservation the structure of the metal-solvent lattice.

According to [25], the physical reason for the formation of such ordered regions is a decrease in the free energy of the system in the formation of segments with an increased concentration of atoms of one class. Such areas can increase as a result of ascending diffusion $[25,26]$. As is shown by the description of alloys near the stoichiometric composition corresponding to the formula $\mathrm{AB}_{3}$ [25], the most fully investigated in this area are alloys of the $\mathrm{AuCu}$ system near the composition of $\mathrm{AuCu}_{3}$. The ordered phase in such systems exists in the range of concentrations from 17 at. \% up to 37 at. \%, which was also revealed for both Pd-In-Ru foils. In atoms in such ordered regions, occupying three times fewer positions, can be located at the vertices of cubic cells, and palladium atoms in the centers of faces.

It should be noted that alloys having a face-centered cubic lattice in an unordered state, upon formation of areas with content of components approaching 50\% composition, can have an ordered state structure of the type $\mathrm{AuCu}$ [25]. Atoms of each kind in this type of ordering are arranged in alternating atomic planes and the crystal lattice becomes tetragonal during the ordering. In the experiment, this type of ordering corresponds superstructural phase PdIn.

Exceptional characteristics of SR [17-19] allowed us to identify fluctuations in the arrangement of atoms in superstructural phases. The presence of domains of different degree ordering in the alloy matrix has led to a nontrivial form of diffraction profiles.

\section{Conclusions}

X-ray structural studies of diffusion filters using SR revealed the existence of long-range ordering regions in both foils.

The facts presented in this paper show the quasistability of superstructural inclusions in membranes in the hydrogenation process.

Analysis of angular positions of the diffraction peaks corresponding to superstructural phases showed that the phases they determine fell into two large structural groups. One of them, the $\mathrm{Pd}_{3} \mathrm{In}$ composition, has a face-centered lattice in both foils, similar to the structure of $\mathrm{AuCu}_{3}$. Another group is the regions of the PdIn composition with an $\mathrm{AuCu}$ type structure.

The exceptional characteristics of the SR made it possible to obtain for the first time in the experimental work the results reflecting the continuum of domains of transition concentrations and fluctuations in the distribution of metal atoms by concentration in the ordering regions.

The work was presented as a plenary (oral) speech at the International Conference "Solid State Chemistry and Functional Materials" (12th International Symposium "Thermodynamics and Materials Science"), held at Ioffe Institute (May 21-27, 2018).

\section{Acknowledgement}

The synchrotron diffraction measurements were performed at unique scientific equipment Kurchatov Synchrotron Radiation source at NRC Kurchatov institute, which partially financially supported by Science and Education Ministry of Russian Federation by the State program "Investigation and development of priority directions of development of Russian scientific and technological complex in 2014-2020", agreement 14.619.21.0007, project RFMEFI61917X0007. 


\section{References}

[1] Grashoff G.J., Pelkington C.E., Corty C.W. The purification of Hydrogen. Platinum Metals Review, 1983, 27(4), P. 157-169.

[2] Stiller C., Schmidt P., Michalski J. Storage of Renewable Electricity through Hydrogen Production. World Renewable Energy Congress. Linköping University Electronic Press. 2011, 15, P. 4202-4208.

[3] Tereshchuk V.S. Hydrogen Generator Based on Reduced Solid Fuel. Russian Engineering Research, 2011, 31(12), P. 1205-1212.

[4] Burkhanov G.S. , Gorina N.B., Kolchugina N.B., Roshan N.R., Slovetsky D.I., Chistov E.M. Palladium-Based Alloy Membranes for Separation of High Purity Hydrogen from Hydrogen-Containing Gas Mixtures. Platinum Metals Review, 2011, 55(1), P. 3-12.

[5] Knyaginichev A.V., Khan KhaSok, Avdyukhina V.M., Katsnel'son A.A., Revkevich G.P. Physics of the evolution of structure and elastic stresses in Pd-Mo alloys saturated with hydrogen. Physics of the Solid State, 2001, 43(2), P. 207-213.

[6] Fukai Y., Okuma N. Formation of Superabundant Vacancies in Pd Hydride under High Hydrogen Pressures. Physical Review Letters, 1994, 73(12), P. 1640-1668.

[7] Dos Santos D.S., Tavares S.M., Miraglia S., Fruchart D., dos Santos D.R. Analysis of the nanopores produced in nickel and palladium by high hydrogen pressure. Journal of Alloys and Compounds, 2003, 356-357, P. 258-262.

[8] Alefeld G., Felkl I. Hydrogen in metals. M.: Mir, 1981, 430 p.

[9] Tawancy H.M. Correlation between disorder-order transformations in a Ni-based alloy and its mechanical properties. Materials Science and Engineering: A, 2018, 719, P. 93-103.

[10] Straumal B.B., Kilmametov A.R., Lopez G.A., Lopez-Ferreno I., No M.L., San Juan J. Hahn H., Batretzky B. High-b pressure torsion driven phase transformations in $\mathrm{Cu}-\mathrm{Al}-\mathrm{Ni}$ shape memory alloys. Acta Materialia, 2017, 125, P. $274-285$.

[11] Avdyukhina V.M., Akimova O.V., Levin I.S., and Revkevich G.P. Nonmonotonic Redistribution of Indium Atoms in Pd-In-Ru Alloy Foils after Electrolytic Hydrogenation. Russian Metallurgy (Metally), 2011, 7, P. 646-651.

[12] Avdyukhina V.M., Akimova O.V., Levin I.S., and Revkevich G.P. The Influence of Hydrogenation on the Redistribution of Indium in a $\mathrm{Pd}-\mathrm{In}-\mathrm{Ru}$ Alloy in the Course of Relaxation. Moscow University Physics Bulletin, 2011, 66(1), P. 33-38.

[13] Avdyukhina V.M., Akimova O.V., Levin I.S., and Revkevich G.P. Changes in the Phase Structure State of Pd-In-Ru Alloy Foils after Hydrogenation and Prolonged Relaxation. Moscow University Physics Bulletin, 2014, 69(4), P. 349-356.

[14] Jamieson H.C., Weatherly G.C., Manchester F.D. The Transformation in Palladium-Hydrogen Alloys. J. Less. Comon. Metals, 1976, 50, P. 85-102.

[15] Avdyukhina V.M., Revkevich G.P., Katsnel'son A.A. Special role of vacancies during structural transformation of Pd-H and Pd-M-H system. J. Alter. Energ. Ecolog., 2005, 7, P. 20-28.

[16] Akimova O.V., Avdyukhina V.M., Shchetinin I.V. Role of vacancies in the relaxation of Pd-5.3 at. \% In-0.5at. \% Ru alloy foil after hydrogen desorption. The Physics of Metal and Metallography, 2016, 117(2), P. 130-134.

[17] Koval'chuk M.V., Kvardakov V.V., Korchuganov V.N., KISR-yesterday, today, tomorrow. Priroda, 2013 , 12, P. $25-36$.

[18] Zubavichus Y.V., Slovokhotov Y.L. X-ray Synchrotron Radiation in physico-chemical studies. Chemical success, $2001,70(5)$, P. 429-463.

[19] Akimova O.V., Veligzhanin A.A. Structural Diagnostics of Metal Alloys-New Possibilities in the Use of Synchrotron Radiation. Journal of Surface Investigation: X-ray, Synchrotron and Neutron Techniques, 2018, 12(1), P. 111-115.

[20] Chernyshov A.A., Veligzhanin A.A., Zubavichus Y.V. Structural Materials Science end-station at the Kurchatov Synchrotron Radiation Source: Recent instrumentation upgrades and experimental results. Nucl. Instrum. Methods Phys. Res. A, 2009, 603(1-2), P. 95-98.

[21] Hammersley A.P. FIT2D: a multi-purpose data reduction, analysis and visualization program. J. Appl. Crystallogr., 2016, 49(2), P. 646-652.

[22] Wojdyr M. Fityk: a general-purpose peak fitting program. J.Appl. Cryst., 2010, 43, P. 1126-1128.

[23] Lyakishev N.I. State diagrams of double metal systems. M.: Mechanical Engineering, 2001, 3(1), 872 p.

[24] Iveronova V.I., Revkevich G.P. Theory of X-ray scattering. Moscow, 1978, 278 p.

[25] Krivoglaz M.A., Smirnov A.A. Theory of ordering alloys. Moscow, 1958, 388 p.

[26] Revkevich G.P., Mitkova M.K., Katsnel'son A.A. Phenomenon of upward diffusion in hydrogen-saturated alloys palladium-samarium. Vestnik Mosk. Univ., 1997, 38(2), P. 30-33. 